\title{
Efecto de la calabaza fermentada (Cucúrbita pepo) en los parámetros productivos y de salud en cerdos en preceba
}

Effect of fermented pumpkin (Cucúrbita pepo) on the productive and health parameters in pre-fattening pigs

Rafael Medina-González ${ }^{1}$

Abel Ortiz-Milán ${ }^{2}$

Arabel Elias-Iglesias ${ }^{3}$

Victor M. Álvarez-Villar ${ }^{4}$

Odelin Brea-Maure ${ }^{5}$

Fecha de recepción: 9 de septiembre de 2018

Fecha de aprobación: 2 de diciembre de 2018

\section{Resumen}

Se utilizaron 30 cerdos en preceba del hibrido Yorkshire $x$ Landrace, de peso promedio $7 \mathrm{~kg}$, durante 42 días, coincidiendo entre los 33 a los 76 días de edad de los cerdos, con el objetivo de sustituir el 0 (control); 15 y $30 \%$ del pienso convencional por el fermentado de calabaza, según diseño completamente aleatorizado con tres tratamientos y diez repeticiones, cada cría se consideró como una repetición. Se determinó la concentración de levaduras en el fermentado de calabaza donde se observó una estabilidad aparente en la concentración de estos microorganismos a los 21 días de elaborado el fermentado, mientras que en los cerdos el peso vivo final (27.30; 28.00 y $27.50 \mathrm{~kg}$ ), la ganancia media diaria (483; 502 y 488 g/día), el consumo de materia seca $(30.37 ; 30.38$ y $30.33 \mathrm{~kg})$ y la conversión de la materia seca (1.50; 1.44 y $1.48 \mathrm{~kg} \mathrm{MS} / \mathrm{kg}$ PV respectivamente); no presentaron diferencia significativa entre los tratamientos evaluados, sin embargo en el tratamiento control hubo mayor cantidad de animales con diarreas y la mortalidad alcanzó el $10 \%$; mientras que en los tratamientos que incluyeron fermentado de calabaza el índice de diarrea fue mínimo y las muertes nula. Se concluyó que el fermentado de calabaza no afectó los indicadores productivos de los cerdos en preceba, al tiempo que reduce las ocurrencias de diarreas y evita las muertes en esta categoría tecnológica.

Palabras clave: alimento fermentado; cerdos; levaduras.

\footnotetext{
${ }^{1}$ M. Sc. Universidad de Guantánamo (Guantánamo, Cuba).

2 Ph. D. Universidad de Guantánamo (Guantánamo, Cuba). abel@cug.co.cu.

${ }^{3}$ Ph. D. Instituto de Ciencia Animal (Mayabeque, Cuba). aelias@ica.co.cu.

${ }^{4}$ Ph. D. Universidad de Guantánamo (Guantánamo, Cuba). mvictor@cug.co.cu.

${ }^{5}$ Ph. D. Universidad de Matanzas Camilo Cienfuegos (Matanzas, Cuba). odelin.brea@umcc.cu. 
Efecto de la calabaza fermentada (Cucúrbita pepo) en los parámetros productivos y de salud en cerdos en preceba

\section{Abstract}

There were used 30 pre-fattening pigs of Yorkshire $x$ Landrace hybrid, $7 \mathrm{~kg}$ average weight, during 42 days, coinciding between 33 to 76 days of the pigs' age, with the objective of replacing the 0 (control); 15 and $30 \%$ of the conventional feed for the fermented pumpkin, according to completely randomized design with three treatments and ten repetitions, each baby was considered as a repetition. The concentration of yeasts in the pumpkin fermentation was determined, where an apparent stability in the concentration of these microorganisms was observed 21 days after the fermented was elaborated, while in the pigs the final live weight $(27.30,28.00$ and $27.50 \mathrm{~kg}$ ), the average daily gain (483, 502 and $488 \mathrm{~g} /$ day), the consumption of dry matter (30.37, 30.38 and $30.33 \mathrm{~kg}$ ) and the conversion of dry matter $(1.50,1.44$ and $1.48 \mathrm{~kg} \mathrm{DM} / \mathrm{kg}$ PV respectively) did not present a significant difference between the treatments evaluated; however, in the control treatment, there were more animals with diarrhea and mortality reached $10 \%$; while in treatments that included fermented pumpkin, the rate of diarrhea was minimal and deaths were nil. It is concluded that pumpkin fermentation did not affect the productive indicators of pre-fattening pigs, while reducing the occurrence of diarrhea and avoiding deaths in this technological category.

Keywords: fermented food; pigs; yeasts.

\section{Para citar este artículo:}

Medina-González, R., Ortiz-Milán, A., Elias-Iglesias, A., Álvarez-Villar, V. M., \& BreaMaure, O. (2019). Efecto de la calabaza fermentada (Cucúrbita pepo) en los parámetros productivos y de salud en cerdos en preceba. Ciencia y Agricultura, 16(1), 79-91. DOI: https://doi.org/10.19053/01228420.v16.n1.2019.8835.

\section{Esta obra está bajo licencia internacional Creative Commons Reconocimiento 4.0}




\section{Introducción}

La creciente demanda de proteína de origen animal con destino al consumo humano, genera gran interés en la producción de animales de rápido crecimiento y corto intervalo generacional (Anya et al., 2011). En este propósito el cerdo es un elemento clave dentro de la producción pecuaria. Esto se debe a su capacidad de adaptación a diferentes sistemas de manejo y alimentación, su alta prolificidad y la variedad de productos que proporciona (Camejo, 2013).

En las condiciones actuales de la producción porcina, donde las pequeñas fincas del campesino ocupan un papel relevante, se hace necesario realizar ajustes en las tecnologías de producción que se han aplicado en etapas anteriores. Estas tecnologías no pueden sustentarse en la importación de cereales, se debe disponer de una base alimentaria nacional que respalde el propósito del Ministerio de la Agricultura para incrementar la producción de carne de cerdo en Cuba de forma significativa en los próximos años (Brea et al., 2014).

En la actualidad se desarrollan diversas investigaciones enfocadas en la alimentación de animales monogastricos, para incrementar el valor nutricional y reducir las limitantes nutricionales de diferentes productos. Una de estas alternativas es por vía de la fermentación, a través de la cual se pueden obtener alimentos energético-proteicos biotransformados en sus diferentes variantes donde se incluyen fuentes energéticas y proteínicas para mejorar la calidad y digestibilidad del producto que se fermenta (Rodríguez et al. 2015; Rodríguez et al. 2013; Ortiz et al., 2017).

Por lo anteriormente expuesto, es necesario conocer el efecto que producen los procesos fermentativos en el valor nutritivo de un determinado alimento ya que este proceso puede incrementar la concentración de microorganismos con potencialidades probióticas y con ellos lograr mejores resultados en los animales que lo consumen (Brea et al., 2015). 
En Cuba, se puede aprovechar los beneficios que brindan cultivos como la calabaza (Cucúrbita pepo). En la provincia de Guantánamo se producen anualmente más de 10000 toneladas, un gran parte de esta son utilizadas directamente por los productores como alimento en cerdos, de ahí que someter a la Cucúrbita pepo a un proceso fermentativo y usarla posteriormente como sustituto parcial del alimento convencional de cerdos en preceba, resultó en el objetivo a tratar en la presente investigación.

\section{Materiales y métodos}

La investigación se realizó en condiciones de producción en un convenio integral porcino del sector privado con experiencia en los procesos fermentativos y donde se aprovechó las bondades de esta técnica en la conservación de la Cucúrbita pepo.

\section{A. Ubicación y alojamiento de los animales}

El trabajo se realizó en la Finca "La Juanica" perteneciente a la Cooperativa de Crédito y Servicio Fortalecida (CCSF) Ángel Bouza Calvo, ubicado en el km 22 de la carretera Guantánamo- Yateras, en el municipio Manuel Tames de la provincia de Guantánamo, Cuba, en el período comprendido de febrero a marzo de 2017.

Se utilizaron 30 cerdos en preceba del hibrido Yorkshire $x$ Landrace, de peso promedio $7 \mathrm{~kg}$, durante 42 días, coincidiendo entre los 33 a los 76 días de edad de los cerdos, y se distribuyeron en un diseño completamente aleatorizado con tres tratamientos y diez repeticiones, cada cría se considero como una repetición y se identificaron con numeración en la oreja derecha.

\section{B. Tratamientos e indicadores}

Una vez concluido el fermentado de calabaza y estando listo este para ser empleado como alimento en los cerditos, se procedió a realizar el estudio de las concentraciones de levadura en el fermentado de calabaza en tres momentos del experimento con los cerdos: I-inicio (1er día) II- Intermedio (21 días) y III-final (42 días), para lo cual se tomaron cinco muestras aleatorias en 
cada momento y así poder determinar posibles variaciones en la concentración de levaduras.

En este sentido se realizaron diluciones seriadas de las muestras $(1: 10, p / v)$ en medio diluyente hasta $10^{-6}$. Las muestras fueron analizadas en el laboratorio de microbiología de la Facultad Agroforestal de la Universidad de Guantánamo, según las normas establecidas para el microorganismo investigado. A los resultados se les realizó análisis de varianza según diseño completamente aleatorizado con tres tratamientos (momentos del experimento) y cinco repeticiones, y para detectar las diferencias entre las medias se utilizó la prueba de rango múltiple de Duncan (1955).

Las dietas evaluadas en los cerditos en preceba consistieron en la sustitución del 0 (control); 15 y 30 \% del pienso convencional por el fermentado de calabaza. Para determinar el efecto del fermentado de calabaza se realizó análisis de varianza a las variables peso de inicio y final de los animales $(\mathrm{kg})$, ganancia de peso $(\mathrm{kg})$, ganancia media diaria $(\mathrm{g})$, consumo $(\mathrm{kg})$ y conversión (kg).

En todos los casos la comparación post hoc de las medias se realizó a través de la prueba de rango múltiple de Duncan (1955). El programa utilizado para el análisis de varianza fue el SPSS 15.0 (IBM, 2009).

Fueron contralados también indicadores referentes a la salud de los cerditos, entre ellos se notificaron los siguientes:

- Diarreas/ días: El control de este indicador se realizo durante todo el periodo de investigación, por animal y por día.

- Frecuencia de Diarrea/ animal: A cada cerdo con desorden digestivo se le realizo el control de las frecuencias de diarreas por días.

- Mortalidad: Número de animales que murieron durante el experimento por tratamiento.

- Morbilidad: Número de animales enfermos por tratamiento.

- Viabilidad: Cantidad de animales vivos al final del experimento. 


\section{Manejo de la alimentación y elaboración del fermentado de calabaza}

Los aportes del pienso convencional utilizado se muestran en la Tabla 1, mientras que la Tabla 2 muestra la composición en materias primas de la calabaza fermentada. Todas estas materias primas que componían el fermentado de calabaza se mezclaron homogéneamente durante 5 minutos y luego se dejaron en reposo por nueve días, tiempo a partir del cual estaba lista par ser utiliza como alimento en los cerdos.

Tabla 1. Composición y aportes del pienso convencional.

\begin{tabular}{|l|c|}
\hline \multicolumn{1}{|c|}{ Materias primas } & Inclusión (\%) \\
\hline Harina de maíz & 57.48 \\
\hline Harina de soya & 39.49 \\
\hline Fosfato dicálcico & 1.00 \\
\hline Carbonato de calcio & 1.13 \\
\hline Sal común & 0,35 \\
\hline Premezcla Vitaminica & 0.45 \\
\hline Colina & 0.10 \\
\hline \multicolumn{2}{|c|}{ Aportes } \\
\hline Materia Seca, \% & 88.00 \\
\hline Proteína bruta, \% & 21.00 \\
\hline Energía Digestible, MJ / Kg & 14.25 \\
\hline Calcio, \% & 0.75 \\
\hline Fósforo total, \% & 0.31 \\
\hline
\end{tabular}

Tabla 2. Composición y aportes de la calabaza fermentada.

\begin{tabular}{|l|c|}
\hline \multicolumn{1}{|c|}{ Materias primas } & \multicolumn{1}{c|}{$\begin{array}{c}\text { Porcentaje de } \\
\text { Inclusión }\end{array}$} \\
\hline Calabaza & 76.60 \\
\hline Melaza & 10.00 \\
\hline Prebiótico vitafer & 3.00 \\
\hline Fosfato de calcio & 0.20 \\
\hline $\begin{array}{l}\text { Premezcla } \\
\text { minerovitaminica }\end{array}$ & 0.20 \\
\hline Harina de soya & 5.00 \\
\hline Harina de maíz Aportes \\
\hline \multicolumn{2}{|c|}{5.00} \\
\hline Materia Seca, \% & 25.00 \\
\hline Proteína bruta, \% & 17.92 \\
\hline $\begin{array}{l}\text { Energía Digestible, MJ / } \\
\text { Kg }\end{array}$ & 14.08 \\
\hline Calcio, \% & 1.40 \\
\hline Fósforo total, \% & 0.90 \\
\hline
\end{tabular}

El fermentado de calabaza se mezcló con el pienso y se aplicó a los cerdos siguiendo el esquema que se muestra en la Tabla 3.

Tabla 3. Consumo diario de alimento por animal por tratamiento.

\begin{tabular}{|c|c|c|c|c|c|c|}
\hline Preceba & $\begin{array}{c}\text { Edades } \\
\text { (días) }\end{array}$ & $\begin{array}{c}\text { Consumo } \\
\text { diario } \\
\text { (Control) }\end{array}$ & \multicolumn{2}{|c|}{$\begin{array}{c}\text { Consumo } \\
\text { diario } \\
\mathbf{1 5 \%} \text { FC }\end{array}$} & \multicolumn{2}{c|}{$\begin{array}{c}\text { Consumo } \\
\text { diario } \\
\mathbf{3 0 \%} \text { FC }\end{array}$} \\
\hline Semana & & Pienso (g) & Pienso (g) & FC (I) & Pienso (g) & FC (I) \\
\hline 1 & $34-41$ & 250 & 212 & 0.3 & 175 & 0.6 \\
\hline 2 & $41-48$ & 490 & 420 & 0.3 & 340 & 0.6 \\
\hline 3 & $48-55$ & 680 & 578 & 0.4 & 476 & 0.8 \\
\hline 4 & $55-62$ & 920 & 782 & 0.4 & 644 & 0.8 \\
\hline 5 & $62-69$ & 1140 & 969 & 0.6 & 798 & 1.2 \\
\hline 6 & $69-76$ & 1450 & 1232 & 0.6 & 1015 & 1.2 \\
\hline Acumulado de alimento, $\mathrm{kg}$ & & 34.51 & 29.35 & 18.2 & 24.13 & 36.4 \\
\hline Acumulado de MS, $\mathrm{kg}$ & $(88 \% \mathrm{MS})$ & $(88 \% \mathrm{MS})$ & $(25 \% \mathrm{MS})$ & $(88 \% \mathrm{MS})$ & $(25 \% \mathrm{MS})$ \\
\hline Consumo total de MS, $\mathrm{kg}$ & 30.37 & 25.83 & 4.55 & 21.23 & 9.10 \\
\hline
\end{tabular}

FC: Fermentado de calabaza 


\section{Resultados y discusión}

En la actualidad para definir un probiótico, se utiliza la definición emitida por la FAO/WHO (2002), que se refiere a microorganismos vivos que al ser administrados en cantidades adecuadas proporcionan o generan efectos benéficos en la salud del huésped. Es importante señalar que estos microorganismos no deben ser patógenos ni producir efectos colaterales adversos. Además, constituyen una alternativa al uso de antibióticos promotores del crecimiento animal.

Según Kim et al., (2012) las concentraciones de microorganismos con efecto probiótico es uno de los factores que puede incidir en la respuesta de los animales. Los microorganismos al ser ingeridos deben resistir la acidez gástrica y las sales biliares, que son las primeras barreras que limitan su supervivencia en el ecosistema gastrointestinal. De ahí que, al suministrar concentraciones superiores, los microorganismos tienen mayores posibilidades de sobrevivir y poder ejercer, posteriormente, su actividad probiótica. Las concentraciones empleadas en el presente estudio (Tabla 4) se encontraron en el rango sugerido por la FAO/WHO (10), quienes plantearon que los probióticos deben tener una concentración de $10^{6}$ a $10^{7}$ células $\mathrm{mL}^{-1} \circ \mathrm{g} \mathrm{g}^{-1}$ para garantizar su eficacia.

Tabla 4. Concentración de levaduras en el fermentado de calabaza en tres momentos del experimento.

\begin{tabular}{|c|c|c|c|c|c|}
\hline \multirow{2}{*}{ Levaduras totales } & \multicolumn{3}{|c|}{ Días de experimento } & \multirow{2}{*}{ EE $\mathbf{2}$} & \multirow{2}{*}{ Sig. } \\
\cline { 2 - 6 } & $\mathbf{1}$ & $\mathbf{2 1}$ & $\mathbf{4 2}$ & 0.42 & $\mathrm{P}<0.05$ \\
\hline Levaduras totales, ufc. $10^{-6} / \mathrm{ml}$ & $8.33^{\mathrm{b}}$ & $10.83^{\mathrm{a}}$ & $10.83^{\mathrm{a}}$ & 0 \\
\hline
\end{tabular}

ab Medias con letras diferentes dentro de la misma fila difieren a $\mathrm{P}<0.05$ (Duncan 1955).

En la Tabla 4 se observó como las levaduras se incrementaron significativamente en la medida que se incrementó el tiempo de elaborado del fermentado de calabaza (Figura 1) en este sentido García (2011) observó como en el producto denominado vitafer con capacidad probiotica también se incrementaba la concentración de microorganismos con efecto probiótico con el paso del tiempo hasta alcanzar la estabilidad en la concentración microbiana, aspecto que al parecer se logra en el fermentado de calabaza a los 21 días de 
elaborado, al no presentar las concentraciones de levadura diferencias significativa con respecto a las concentraciones encontradas a los 42 días.
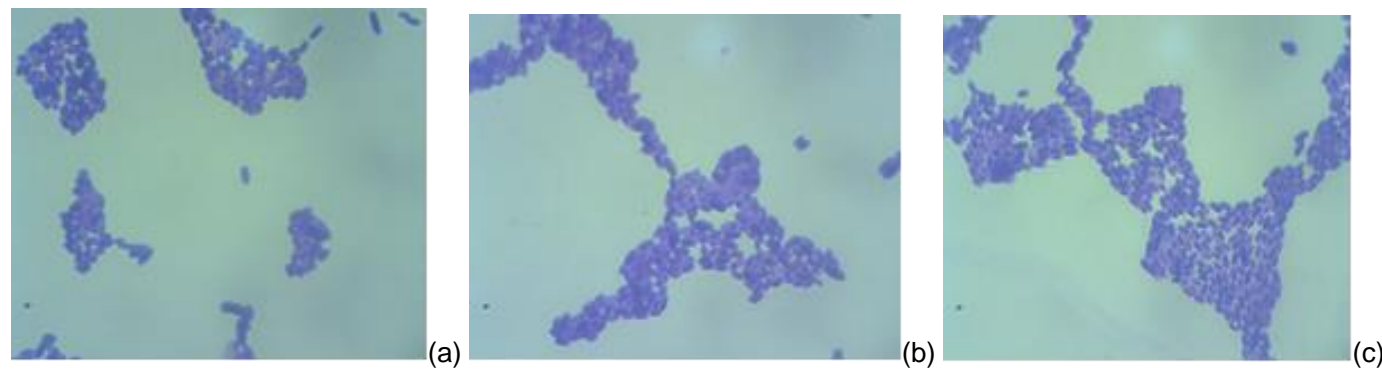

Fig. 1. Concentración de levadura: (a) 1 día, (b) 21 días, y, (c) 42 días.

La alimentación del cerdo recién destetado es uno de los aspectos más críticos en las explotaciones porcinas y supone todo un reto para los nutricionistas y formuladores. La dieta que se suministre al lechón debe ser de excelente calidad nutricional y minimizar el estrés al destete, lo que le permitirá al cerdo, que es separado de su madre en un estadio temprano de su vida, poder desarrollarse adecuadamente en su nuevo ambiente (Pluske et al., 2003).

La Tabla 5 muestra como al sustituir el 15 y el $30 \%$ del pienso convencional por el fermentado de calabaza, no se afectaron los indicadores productivos de los cerdos en preceba, lo cual indica que aún cuando en las dietas investigadas aportaran menos cantidad de proteína bruta que el control, estas por su fácil digestibilidad y concentraciones en levaduras, ejercieran un efecto positivo en la digestibilidad y absorción de los nutrientes en el tracto digestivo de los cerditos, lo cual promovió excelentes ganancias de peso vivo diario y final, lo que a su vez estimuló la conversión de la MS.

Tabla 5. Comportamiento productivo de los animales empleados en la etapa de preceba con la inclusión de la dieta de calabaza fermentada.

\begin{tabular}{|l|c|c|c|c|c|}
\hline \multirow{2}{*}{ Indicadores } & \multicolumn{3}{c|}{ Nivel de calabaza fermentada } & \multirow{2}{*}{ EE $\mathbf{~}$} & \multirow{2}{*}{ Sig. } \\
\cline { 2 - 4 } & $\mathbf{0} \%$ Control & $\mathbf{1 5} \%$ & $\mathbf{3 0} \%$ & & \\
\hline Peso Inicial, Kg & 7.00 & 6.90 & 7.00 & 0.04 & $\mathrm{P}=0.16$ \\
\hline Peso Final, Kg & 27.30 & 28.00 & 27.50 & 0.22 & $\mathrm{P}=0.34$ \\
\hline Ganancia de Peso, Kg & 20.30 & 21.10 & 20.50 & 0.33 & $\mathrm{P}=0.28$ \\
\hline Ganancia Media Diaria, g/día & 483 & 502 & 488 & 6.90 & $\mathrm{P}=0.52$ \\
\hline Consumo alimento, Kg & $34.50^{\mathrm{c}}$ & $47.55^{\mathrm{b}}$ & $60.53^{\mathrm{a}}$ & 3.40 & $\mathrm{P}<0.05$ \\
\hline Consumo MS, Kg & 30.37 & 30.38 & 30.33 & 0.07 & $\mathrm{P}=0.39$ \\
\hline Conversión, Kg alimento/Kg PV & $1.70^{\mathrm{c}}$ & $2.25^{\mathrm{b}}$ & $2.95^{\mathrm{a}}$ & 0.09 & $\mathrm{P}<0.05$ \\
\hline Conversión, Kg MS /Kg PV & 1.50 & 1.44 & 1.48 & 0.05 & $\mathrm{P}=0.31$ \\
\hline
\end{tabular}

abc Medias con letras diferentes dentro de la misma fila difieren a $\mathrm{P}<0.05$ (Duncan 1955). 
El consumo total y la conversión del alimento en base húmeda se incrementaron con 15 y 30 \% de calabaza fermentada con respecto al control, debido al elevado contenido en húmeda de este alimento fresco, sin embargo, con respecto a la materia seca total consumida fue prácticamente igual entre los tres tratamientos y no existió diferencias significativas entre ellos.

En investigaciones anteriores, Brea et al., (2014) al estudiar diferentes niveles de inclusión $(0,10,15$ y 20\%) de harina de frutos del árbol del pan (HFP) sin fermentar, determinaron como límite máximo, hasta un 15\%, sin provocar cambios en los indicadores productivos como sustituto parcial de la harina de maíz en dietas para cerdos en preceba. Sin embargo, al incorporar $20 \%$ de HFP, disminuyó el peso vivo final, GMD y empeoró la conversión alimenticia, con respecto al control.

Más tarde esta autora en el 2015 fermentó la harina del fruto del árbol del pan y logró incluirla en cerdos en preceba hasta niveles del $25 \%$ sin alterar el peso final de los animales, el consumo y la conversión; lo cual demuestra la factibilidad productiva de fermentar alimentos para cerdos recién destetados.

Los probióticos, también, pueden crear temporalmente un microambiente favorable para que crezcan otros microorganismos intestinales y que se produzca una respuesta de tipo probiótica (Balcázar et al., 2006). Tal es el caso de las levaduras que sintetizan vitaminas del complejo $B$, las que estimulan el crecimiento de las poblaciones de Lactobacillus y bacterias acetogénicas y provoca una acción sinérgica y efectos beneficiosos para el huésped (Yoo et al., 1997; Rodríguez, 2011).

Las levaduras, como se conoce, también se emplean en preparaciones probióticas y sus efectos pueden depender de la cepa o especie evaluada. Según Castro y Rodríguez (2005) las levaduras no colonizan el tracto digestivo, pero pueden estimular las disacaridasas de las microvellosidades del tracto gastrointestinal, estimular la inmunidad innata e inducir efectos antiadhesivos y 
antagonistas frente a patógenos, que se traducen en la mejora de los rendimientos productivos de especies monogástricas. Probablemente, las levaduras presentes en el fermentado de calabaza ejercieran estas acciones e incidieran positivamente en la respuesta productiva de los cerditos, así como en la reducción de las diarreas.

En este sentido las diarreas presentadas por los cerditos en los tratamientos evaluados se muestran en la Tabla 6 , donde se observó el efecto beneficioso de la calabaza fermentada, quien no promovió muertes durante la preceba y apenas un solo animal con diarrea en el tratamiento de $15 \%$ de calabaza fermentada, siendo nulo este padecimiento con $30 \%$ de calabaza fermentada. Sin embargo, en el control se presentaron 4 animales con diarrea durante los primeros 15 días de experimento y 2 en la segunda quincena, con una frecuencia de tres diarreas al día por animal para ambas etapas, lo que arrojó al final del experimento la muerte de un animal.

Tabla 6. Diarreas presentadas por los animales durante la preceba.

\begin{tabular}{|l|c|c|c|}
\hline \multicolumn{1}{|c|}{ Indicadores } & \multirow{2}{*}{ Control } & \multicolumn{2}{c|}{ Calabaza Fermentada } \\
\cline { 3 - 4 } & & $\mathbf{1 5} \%$ & $\mathbf{3 0} \%$ \\
\hline Cantidad de animales & 10 & 10 & 10 \\
\hline Animales con diarrea (Primeros 15 días de experimento) & 4 & 1 & 0 \\
\hline Animales con diarrea (16 a 30) días de experimento) & 2 & 0 & 0 \\
\hline Animales con diarrea (31 a 42 días de experimento) & 0 & 0 & 0 \\
\hline Frecuencia de la diarrea & 3 & 1 & 0 \\
\hline Morbilidad, \% & 40.00 & 5.55 & 0 \\
\hline Mortalidad, \% & 10.00 & 0.00 & 0 \\
\hline Viabilidad, \% & 90.00 & 100 & 100 \\
\hline Muertes, no. de animales & 1 & 0 & 0 \\
\hline
\end{tabular}

Los apuntes de Lalles et al. (2011) corroboran los resultados alcanzados en la presente investigación cuando afirma que el destete impone un gran estrés en lechones y se acompaña de cambios fisiológicos, microbiológicos e inmunológicos en el tracto gastrointestinal. Debido a estos cambios, el período después del destete se caracteriza por una alta incidencia de trastornos intestinales con diarrea y bajo rendimiento del crecimiento de los animales.

Autores como Cortés y Gómez (2011); Rodríguez et al. (2013) y Rondón et al. (2013) utilizaron en cerdos recién destetados preparados con bacterias lácticas 
y levaduras y lograron disminuir los trastornos intestinales como diarrea y bajo rendimiento del crecimiento de los animales. Los autores atribuyeron este efecto beneficioso, a las posibilidades de los microorganismos a mejorar la salud intestinal de los animales, modular su sistema inmune y, por ende, incidir de forma favorable en los rendimientos productivos, con ventajas económicas.

\section{Conclusiones}

El fermentado de calabaza resultó una forma práctica y viable para la conservación eficiente de este alimento alternativo, así como se logró mejorar la calidad nutritiva del fermentado suministrado, lográndose que su aprovechamiento y absorción se hicieran más eficientes. Se promovió además en los cerdos un mayor desarrollo y eficiencia productiva con una significativa reducción del uso de alimentos convencionales.

El uso del fermentado de calabaza en cerdos en preceba no afectó los indicadores productivos de los cerdos en crecimiento, al tiempo que reduce las ocurrencias de diarreas y evita las muertes en esta categoría tecnológica.

\section{Referencias}

Anya, M. I., Ayuk, A. A., Umoren, E. P., Akpojovwo, V. A., \& Ubua, J. (2011). Performance of weaned rabbit fed graded levels of African yam bean in cassava peel meal based diets. Continental $\mathrm{J}$. Agricult. Sci., 5 (2), 25-30.

Balcázar, J. L., De Blas, I., Ruiz-Zarzuela, I., Vendrell, D., Cunningham, D., \& Muzquiz, J. L. (2006). The role of probiotics in aquaculture. Veterinary Microbiology, 114, 173-186. DOI: https://doi.org/10.1016/j.vetmic.2006.01.009.

Brea, O., Elías, A., Ortiz, A., Herrera, F., \& Motta, W. (2014). Utilización de la harina de frutos del árbol del pan (Artocarpus altilis) fermentada en estado sólido, en dietas destinadas a cerdos en preceba. Rev. Cubana de Cien. Agríc., 48(4), 391-398.

Brea, O. (2015). Obtención de un alimento energético-proteico a partir de la fermentación en estado sólido de la harina de frutos del árbol del pan y su empleo dietas para conejos y cerdos (Artocarpus altilis). Tesis Doctoral. Instituto de Ciencia Animal, Mayabeque, Cuba.

Camejo, A. I. (2013). Comportamiento reproductivo en cerdas de la UEB Cuba Sí 3 del municipio Holguín. Tesis de Maestría. Instituto de Ciencia Animal, Mayabeque, Cuba.

Castro, M., \& Rodríguez, F. (2005). Levaduras: probióticos y prebióticos que mejoran la producción animal. Revista Corpoica, 6, 26-38. DOI: https://doi.org/10.21930/rcta.vol6 num1 art:33.

Cortés, L., \& Gómez, F. (2011). Efficiency of microorganisms (EM) in the functional improvement of the digestive system of pigs in post-weaning phase. Revista Spei Domus., 25, 31-34.

Duncan, D. B. (1955). Multiple ranges and multiple F test. Biometrics, 11(1), 1-42. DOI: https://doi.org/10.2307/3001478.

$\mathrm{FAO} / \mathrm{WHO}$ (2002). Report of a joint $\mathrm{FAO}$ WHO working group on drafting guidelines for the evaluation of probiotics in food. Ontario, Canada. Disponible en: http://www.who.int/foodsafety/fs management/en/probiotic guidelines.pdf.

García, Y. (2011). Obtención de microorganismos con actividad probiótica a partir de excretas de pollos de ceba fermentadas. Tesis doctoral. Instituto de Ciencia Animal, Mayabeque, Cuba.

IBM (2009). Statistical Package for Social Sciences. New York. 
Efecto de la calabaza fermentada (Cucúrbita pepo) en los parámetros productivos y de salud en cerdos en preceba

Kim, E., Hong, H., Hong, N., Choi, K., Hann, Y., Kangwan, N., Chao, Y., \& Hahn, K. (2012). Concentrated Probiotics Improve Inflammatory Bowel Diseases Better that Commercial Concentration of Probiotics. Journal of Food and Drug Analysis, 20, 292-295.

Lalles, J., Boudry, G., Favier, C., Le Floc'h, N., Lurona, I., Montagne, L., Oswald, I. P., Pie, S., Piel, C., \& Seve, B. (2004). Gut function and dysfunction in young pigs: physiology. Animal Research, 53, 301316. DOI: https://doi.org/10.1051/animres:2004018.

Ortiz, A., Gómez, S., Jay, O., \& Brea, O. (2017). Inclusión del yogurt artesanal de leche de búfala en el pienso de gallinas ponedoras Isa Brown y su efecto en la producción y calidad del huevo. Ciencia y Agricultura, 14(1), 85-93. DOI: https://doi.org/10.19053/01228420.v14.n1.2017.6091.

Pluske, J., Hopwood, D., \& Hampson, D. (2003). Relación entre la microbiota intestinal, el pienso, la incidencia de diarreas y su influencia sobre la salud del lechón tras el destete. En XIX Curso de Especialización FEDNA. Madrid, España.

Rodríguez, B. (2011). Levadura torula desarrollada sobre vinaza de destilerías para la alimentación de aves. Tesis doctoral. Instituto de Ciencia Animal, Mayabeque, Cuba.

Rodríguez, H. C., Barreto, G., Bertot, A., \& Vázquez, O. (2013). Los microorganismos eficientes como promotores del crecimiento en los cerdos hasta el destete. REDVET. Revista Electrónica de Veterinaria, 14, 1-7.

Rodríguez, O., Martín, Y., García, A., \& Núñez, M. (2015). Suero fermentado por lactobacillus casei y lactobacillus acidophilus con propiedades probióticas para cerdos jóvenes. En: V Congreso de Producción Animal Tropical. Palacio de las Convenciones, La Habana, Cuba.

Rondón, A., Ojito, Y., Arteaga, F., Laurencio, M., Milián, G., \& Pérez, Y. (2013). Efecto probiótico de Lactobacillus salivarius $\mathrm{C65}$ en indicadores productivos y de salud de cerdos lactantes. Revista Cubana de Ciencia Agrícola, 47, 401-407.

Yoo, I., Chang, H., Lee, E., Chang, Y., \& Moon, S. (1997). Effect of B vitamin supplementation on lactic acid production by L. casei. Journal Fermentation Bioengineering, 84, 172-175. DOI: https://doi.org/10.1016/S0922-338X(97)82551-2. 